\title{
Method for Calculation of the Current Concentration of Alkali in the Electrolyte During the Water Electrolysis Process
}

\author{
Andrii Rusanov, Victor Solovey, Mykola Zipunnikov*, Vitaliy Semikin \\ A.M. Pidhorny Institute of Mechanical Engineering Problems of NASU, 2/10, Pozharsky str., 61046,
} Kharkiv, Ukraine

zipunnikovnn@ukr.net

Keywords: water electrolysis, electrolyte, alkali concentration, electrical conductivity.

The article proposes a method for calculation of the current concentration of alkali in the electrolyte, taking into account the consumption and replenishment of feed water in the electrolyzer, which allows to estimate the specific electrical conductivity of the electrolyte during electrolysis process. This is important to increase the efficiency of the water electrolysis process. The calculated change of the current concentration of alkali in the electrolyte in high-pressure electrolyzers taking into account the volume of produced hydrogen is given. With the usage of the proposed method, it is established that the current concentrations of alkali in the electrolyte during the operation of the developed highpressure electrolyzers are in the range of optimal concentrations, where the specific electrical conductivity of the electrolyte is close to maximum and changes according to alkali concentration change.

\section{Introduction}

To achieve the global goal of reducing exhaust emissions into the atmosphere, hydrogen is taken as the main alternative to carbon. Decomposition of water into hydrogen and oxygen by electrolysis is a key solution for decarbonization of the environment. Electrolysis of water with the usage of renewable energy sources (sun, wind) makes it possible to minimize energy consumption and emissions for green hydrogen obtaining. This allows us to consider the electrolysis of water as a promising method of hydrogen production for transport and zero emission power plants [1-11].
Alkaline electrolyzers are widely used for the production of green hydrogen with the use of renewable energy sources $[1,3,7-9]$. During the operation of electrolysis plants, the concentration of alkali in the electrolyte changes due to the fact that the electrolysis consumes water, which is part of the electrolyte, and the alkali contained in the solution serves only to transfer ions. After consuming part of water from the electrolyte, it is periodically replenished. Replenishment of feed water is carried out upon reaching the boundary minimum volume of electrolyte. 
The calculated determination of the change in the current concentration of alkali during the operation of electrolysis plants is an important component of the electrolyte specific electrical conductivity assessment to determine the efficiency of water electrolysis.

The purpose of this paper is to develop a method for calculation of the current concentration of alkali in the electrolyte, taking into account the consumption and replenishment of feed water in the electrolyzer.

\section{Selected model and calculation methodology}

During the consumption and periodic replenishment of feed water in the alkaline electrolyzer, the electrolysis process occurs at a variable concentration of alkali in the electrolyte. This leads to a change in the specific electrical conductivity of the electrolyte (Fig. 1, 2), which affects energy consumption during the production of hydrogen and oxygen.

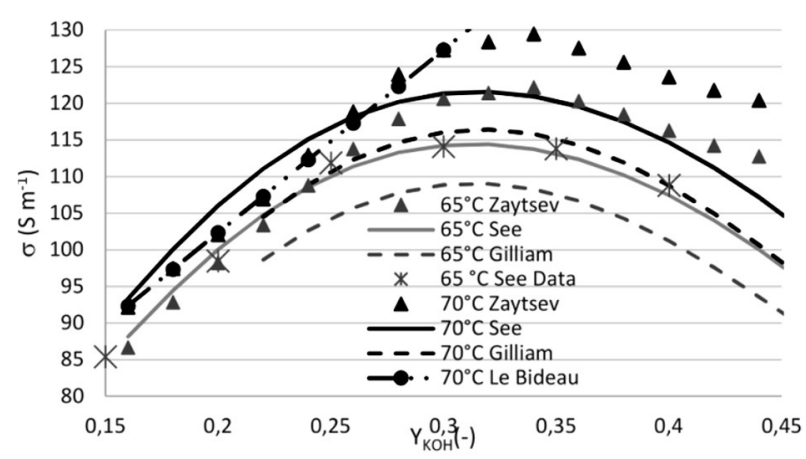

Figure 1. Dependence of specific electrical conductivity of $\mathrm{KOH}$ aqueous solutions on alkali concentration [12].

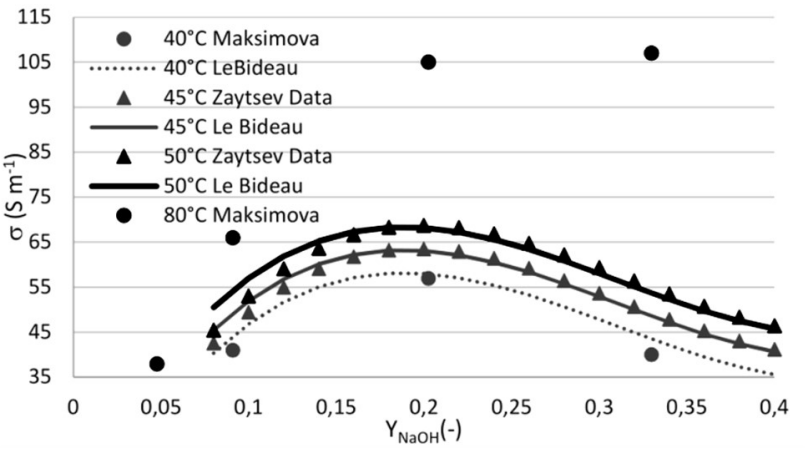

Figure 2. Dependence of specific electrical conductivity of $\mathrm{NaOH}$ aqueous solutions on alkali concentration [12].

The technique of calculation of parameters of feed water replenishment in the alkaline electrolyzer, taking into account the set optimum concentrations of alkali and real expense of electrolyte, is proposed in the article [13]. The formula for calculation of current concentration of alkali in electrolyte under the condition of known productivity of the electrolyzer by hydrogen and production time of consumable part of water during electrolysis is offered.

To obtain a generalized formula for calculated change in the current concentration of alkali in the electrolyte during the design and operation of alkaline electrolysis plants, we use the artificial method proposed in [13], according to which the amount of water in the electrolyte, which fills the electrolysis plant, is divided into two components:

- technological part, which is a technologically necessary minimum volume of water that ensures the functioning of the electrolyzer, i.e. the process of electrolysis;

- consumable part, which is water that decomposes in the process of electrolysis to 
obtain hydrogen and oxygen during the operation of the electrolyzer without replenishment.

Then the mass of water in the electrolyte

$$
\mathrm{m}_{\mathrm{w}}=\mathrm{m}_{\mathrm{wt}}+\mathrm{m}_{\mathrm{wcons}} \text {, }
$$

where $m_{w}$ is the mass of water in the electrolyte; $\mathrm{m}_{\mathrm{wt}}$ is the mass of the technological part of water in the electrolyte; $m_{w c o n s}$ is the mass of the consumable part of water in the electrolyte.

The mass of the electrolyte that fills the electrolysis plant is defined as

$$
\mathrm{m}_{\mathrm{el}}=\mathrm{m}_{\mathrm{w}}+\mathrm{m}_{\mathrm{alk}},
$$

where $m_{\mathrm{el}}$ is the mass of the electrolyte in the electrolysis plant; $m_{\text {alk }}$ is the mass of alkali in the electrolyte of the electrolysis plant.

The concentration of alkali in the electrolyte is defined as

$$
\mathrm{C}_{\mathrm{alk}}=\frac{\mathrm{m}_{\mathrm{alk}}}{\mathrm{m}_{\mathrm{el}}},
$$

where $\mathrm{C}_{\text {alk }}$ is the concentration of alkali in the electrolyte.

From (1), (2) and (3) we get

$$
\mathrm{m}_{\mathrm{el}}=\frac{\mathrm{m}_{\mathrm{w}}}{1-\mathrm{C}_{\text {alk }}}=\frac{\mathrm{m}_{\mathrm{wt}}+\mathrm{m}_{\mathrm{wcons}}}{1-\mathrm{C}_{\text {alk }}} .
$$

In the process of electrolysis, the water that is part of the electrolyte is consumed, and the concentration of alkali in the electrolyte increases from the initial value of $\mathrm{C}_{\text {init }}$ (at the initial moment after replenishment) to the maximum value of $\mathrm{C}_{\max }$ (after the production of all consumable water). The mass of alkali remains almost unchanged, and the mass of water in the electrolyte decreases to the mass of the technological part of water $-m_{w}$. Given this and taking into account (4) the mass of the technologically necessary part of the electrolyte is defined as

$$
\mathrm{m}_{\mathrm{el.t}}=\frac{\mathrm{m}_{\mathrm{wt}}}{1-\mathrm{C}_{\mathrm{max}}} .
$$

where $m_{\text {el.t }}$ is the mass of the technological part of the electrolyte; $\mathrm{C}_{\max }$ is the concentration of alkali in the electrolyte after the production of all consumable water.

Then, given (5), the mass of alkali in the electrolyte

$$
\mathrm{m}_{\mathrm{alk}}=\mathrm{m}_{\mathrm{el.t}} \mathrm{C}_{\max }=\mathrm{m}_{\mathrm{wt}} \frac{\mathrm{C}_{\max }}{1-\mathrm{C}_{\max }} .
$$

From (1), (2), (4) and (6), and taking into account the initial concentration of alkali in the electrolyte, we obtain

$$
\begin{gathered}
m_{\mathrm{el}} \frac{\mathrm{m}_{\mathrm{wt}}+\mathrm{m}_{\mathrm{wcons}}}{1-\mathrm{C}_{\text {init }}}, \\
\left.\mathrm{m}_{\mathrm{el}}=\mathrm{m}_{\mathrm{wt}}\left(1+\frac{\mathrm{C}_{\max }}{1-\mathrm{C}_{\max }}\right)+\mathrm{m}_{\mathrm{wcons}}\right),
\end{gathered}
$$

where $\mathrm{C}_{\text {init }}$ is the concentration of alkali in the electrolyte at the initial moment of operation of the electrolyzer or after replenishment of feed water.

Electrolysis requires $0.820-0.850 \mathrm{~kg}$ of water, which is a part of the electrolyte, to obtain $1 \mathrm{~m}^{3}$ of hydrogen and $0.5 \mathrm{~m}^{3}$ of oxygen [14]. That is, through the amount of hydrogen obtained, one can determine the amount of water consumed during electrolysis

$$
\mathrm{m}_{\mathrm{wcons}}=\mathrm{V}_{\mathrm{H}} \cdot \gamma_{\mathrm{cons}},
$$

where $\mathrm{V}_{\mathrm{H}}$ is the volume of obtained hydrogen; $\gamma_{\text {cons }}$ is feed water consumption to obtain $1 \mathrm{~m}^{3}$ of hydrogen [14].

Using the system of equations (7) and formula (8), replacing $\mathrm{C}_{\max }$ with $\mathrm{C}_{i}$ $\left(\mathrm{C}_{\text {init }} \leq \mathrm{C}_{i} \leq \mathrm{C}_{\max }\right)$, we determine the current 
concentration of alkali in the electrolyte during the production of consumable water by electrolysis

$$
\mathrm{C}_{i}=\frac{1}{\frac{\mathrm{m}_{\mathrm{wt}}}{\left(\mathrm{m}_{\mathrm{wt}}+\mathrm{v}_{\mathrm{H} i} \cdot \gamma_{\mathrm{ccons}}\right) \cdot\left(\frac{1}{1-\mathrm{C}_{\text {init }}}-1\right)}+1},
$$

where $\mathrm{C}_{i}$ is the current concentration of alkali in the electrolyte during the water electrolysis; $\mathrm{V}_{\mathrm{H} i}$ is the volume of hydrogen produced after replenishment of feed water, or the volume of hydrogen calculated by the formula

$$
\mathrm{V}_{\mathrm{H} i}=\tau_{i} \cdot \mathrm{P}_{\mathrm{H} 2},
$$

where $\tau_{i}$ is the current duration of the electrolyzer operation without replenishment of feed water; $\mathrm{P}_{\mathrm{H} 2}$ is performance of the electrolyzer for hydrogen.

Taking into account (10) and replacing $\mathrm{C}_{\text {init }}$ by $\mathrm{C}_{\min }$, we get the formula for calculation of the current concentration of alkali in the electrolyte during water electrolysis, which is proposed in [13].

Thus, the obtained dependence (9) allows to use two options for calculation of the current concentration of alkali in the electrolyte - taking into account the produced hydrogen or based on the performance of the electrolyzer by hydrogen.

In the first option for the calculation of $\mathrm{C}_{i}$ by formula (9):

1.1) the amount of technological part of water in the electrolyte $\mathrm{m}_{\mathrm{wt}}$ is set according to the design of the electrolyzer;

1.2) the initial concentration of alkali in the electrolyte $\mathrm{C}_{\text {init }}$ (set during the initial preparation of the electrolyte for the initial filling of the electrolyzer or measured after replenishment of feed water) is determined;

1.3) the consumption of feed water to obtain $1 \mathrm{~m}^{3}$ of hydrogen $\gamma_{\text {cons }}$ for a specific electrolysis plant is set;

1.4) the amount of total hydrogen $\mathrm{V}_{\mathrm{Hi}}$ produced from the beginning or after replenishment of feed water to the current moment of operation of the electrolyzer (specified during development or measured for the existing electrolyzer) is determined.

In the second option for the calculation of $\mathrm{C}_{i}$ by formula (9) the first three points coincide with the first three points of the previous option: 2.1) the amount of technological part of water in the electrolyte $m_{w t}$ is set according to the design of the electrolyzer;

2.2) the initial concentration of alkali in the electrolyte $C_{\text {init }}$ (set during the initial preparation of the electrolyte for the initial filling of the electrolyzer or measured after replenishment of feed water) is determined;

2.3) the consumption of feed water to obtain $1 \mathrm{~m}^{3}$ of hydrogen $\gamma_{\text {cons }}$ for a specific electrolysis plant is set;

2.4) the performance of the electrolyzer $\mathrm{P}_{\mathrm{H} 2}$ for hydrogen is set or determined (during all operation or in different operation modes of the electrolyzer);

2.5) the current duration of operation of the electrolyzer without replenishment of feed water $\tau_{i}$ (set or measured) is determined.

Based on these data, you can use the formula (9) to calculate the current 
concentrations of alkali in the electrolyte for both research and operational tasks.

\section{Results and their discussion}

The obtained calculated dependence allows to calculate the current concentration and range of changes in the concentration of alkali in the electrolyte both during the development and operation of existing alkaline electrolyzers.

In the first case of calculation of the current concentration of alkali in the electrolyte one does not need to take into account the operation modes of the electrolyzer or its stop. This option is acceptable for both electrolysis plants under development and for existing ones.

The second option for calculation of the current concentration of alkali in the electrolyte can be used in the development of new electrolyzers. This is based on the fact that the operation modes of the electrolyzer, namely changes in the performance of the electrolyzer for hydrogen during operation of the existing electrolyzer are difficult to determine. In particular, it is especially difficult to determine the operation of the electrolyzer in combination with renewable energy sources, which are characterized by instability of electricity generation.

The application of the obtained dependence for alkaline electrolyzers with periodic replenishment of feed water under development and for existing electrolyzers provides an opportunity to analyze the nature of changes in the specific electrical conductivity of the electrolyte, i.e. to evaluate the efficiency of the electrolysis process. This will improve alkaline electrolysis plants and provide additional information for the development and configuration of feed water supply systems to maintain the optimal concentration of alkali in the electrolyte.

For the production of hydrogen and oxygen in the membraneless high-pressure electrolysis plants developed by IPMach NAS of Ukraine [15, 16], $\mathrm{KOH}$ aqueous solutions are used as electrolytes. During operation, electrolyzers require periodic replenishment of feed water consumed from the electrolyte. To ensure the maximum specific electrical conductivity of the electrolyte and taking into account the design features of the developed electrolyzers, the range of changes in the concentrations of $\mathrm{KOH}$ in the electrolyte was selected $25-30 \%$ [13].

Table 1 shows the operational parameters of the high-pressure electrolyzers EHP 1.0-150 and EHP 0.5-150 developed in IPMach NAS of Ukraine and the demonstration model of the high-pressure electrolyzer DM-0.002-3.

Table 1. Operational parameters of high-pressure electrolyzers developed in IPMash NAS of Ukraine.

\begin{tabular}{|c|c|c|c|l|}
\hline Electrolyzer & $\begin{array}{c}\mathrm{P}_{\mathrm{H} 2}, \\
\mathrm{~m}^{3} / \mathrm{h}\end{array}$ & $\begin{array}{c}\mathrm{m}_{\mathrm{wt}}, \\
\mathrm{kg}\end{array}$ & $\begin{array}{c}\gamma_{\text {cons, }} \\
\mathrm{kg} / \mathrm{m}^{3}\end{array}$ & $\mathrm{C}_{\text {init }}$, \\
\cline { 1 - 3 } EHP 1.0-150 & 1.0 & 142.0 & & \\
\cline { 1 - 3 } EHP 0.5-150 & 0.5 & 71.0 & \multirow{2}{*}{0.82} & \multirow{2}{*}{0.25} \\
\cline { 1 - 3 } DM-0.002-3 & 0.002 & 1.7 & & \\
\hline
\end{tabular}


Table 2 shows the calculated change in the current concentration of $\mathrm{KOH}$ in the electrolyzers EHP 1.0-150, EHP 0.5-150 and DM-0.002-3, taking into account the volume of hydrogen produced after replenishment of feed water.

Table 2. The change of the current concentration of $\mathrm{KOH}$ in electrolyzers EHP 1.0-150, EHP 0.5-150 and DM-0.002-3.

\begin{tabular}{|c|c|c|}
\hline Electrolyzer & $\begin{array}{c}\mathrm{V}_{\mathrm{Hi}}, \\
\mathrm{m}^{3}\end{array}$ & $\begin{array}{c}\mathrm{C}_{i}, \\
\%\end{array}$ \\
\hline \multirow{4}{*}{ EHP 1.0-150 } & 1.3 & 25.14 \\
\cline { 2 - 3 } & 2.1 & 25.27 \\
\cline { 2 - 3 } & 5.4 & 25.58 \\
\cline { 2 - 3 } & 11.9 & 26.27 \\
\cline { 2 - 3 } & 18.2 & 26.92 \\
\hline \multirow{4}{*}{ EHP 0.5-150 } & 0.6 & 25.13 \\
\cline { 2 - 3 } & 2.4 & 25.52 \\
\cline { 2 - 3 } & 7.3 & 26.55 \\
\cline { 2 - 3 } & 9,8 & 27.06 \\
\cline { 2 - 3 } & 12.3 & 27.57 \\
\hline \multirow{3}{*}{ DM-0.002-3 } & 0.0007 & 25.006 \\
\cline { 2 - 3 } & 0.0016 & 25.014 \\
\cline { 2 - 3 } & 0.0029 & 25.261 \\
\hline
\end{tabular}

Table 2 data show that the current concentrations of $\mathrm{KOH}$ during the operation of the electrolyzers EHP 1.0-150, EHP 0.5-150 and DM-0.002-3 are in the range of optimal concentrations, where the specific electrical conductivity of the electrolyte is close to the maximum and does not change much when the alkali concentration changes (Fig. 1).

Thus, the calculated determination of the current concentration of alkali during the operation of electrolysis plants allows to assess, in particular with help of [12], the specific electrical conductivity of the electrolyte, which is important for improving the efficiency of water electrolysis.

\section{Conclusions}

Under the conditions of consumption and periodic replenishment of feed water, the process of electrolysis in the alkaline electrolyzer occurs at a variable concentration of alkali in the electrolyte, which leads to changes in the specific electrical conductivity of the electrolyte and affects energy consumption during the production of hydrogen and oxygen.

The proposed dependence allows to determine changes in the current concentration of alkali and, taking them into account, to analyze changes in the specific electrical conductivity of the electrolyte during electrolysis. This provides additional opportunities to increase the efficiency of the process of water electrolysis with alkaline electrolyzers and improve the feed water replenishment systems to ensure maximum specific electrical conductivity of the electrolyte.

The calculation of the current concentration of alkali in the electrolyte showed that the range of changes in the concentration of alkali during the operation of the developed highpressure electrolyzers is in the range of optimal concentrations, where the specific electrical conductivity of the electrolyte is close to maximum. This demonstrates the effectiveness of the proposed method. 


\section{References}

[1] Miller H, Bouzek K, Hnat J, Loos S, Bernäcker C, Weißgärber T, Röntzsch L, Meier-Haack J. Green hydrogen from anion exchange membrane water electrolysis: a review of recent developments in critical materials and operating conditions. Sustainable Energy \& Fuels 2020;4(5):2114-2133.

[2] Rozzi E, Minuto F, Lanzini A, Leone P. Green Synthetic Fuels: Renewable Routes for the Conversion of Non-Fossil Feedstocks into Gaseous Fuels and Their End Uses. Energies 2020;13(2):420.

[3] IRENA (2020), Green Hydrogen Cost Reduction: Scaling up Electrolysers to Meet the $1.5^{\circ} \mathrm{C}$ Climate Goal, International Renewable Energy Agency, Abu Dhabi.

[4] Green Hydrogen from Water Electrolysis, Solution for Sustainability. Energy Industry Review. July 1, 2020.

[5] Renee Cho. Why We Need Green Hydrogen. Columbia Climate School January 7, 2021.

[6] Duke researchers boost electrolyzer productivity with microfibrous flow-through electrode; 12.5 - to 50-times greater than conventional. Green Car Congress. 01 June 2020.

[7] Colli A, Girault H, Battistel A. Non-Precious Electrodes for Practical Alkaline Water Electrolysis. Materials 2019;12(8):1336.

[8] Yang F, Kim M, Brown M, Wiley B. Alkaline Water Electrolysis at $25 \mathrm{~A} \cdot \mathrm{cm}^{-2}$ with a Microfibrous Flowthrough Electrode. Advanced Energy Materials 2020;10(25):2001174.

[9] Ulleberg O, Hancke R. Techno-economic calculations of small-scale hydrogen supply systems for zero emission transport in Norway. International Journal of Hydrogen Energy. 2020;45(2):1201-1211.

[10] Li D, Park E, Zhu W, Shi Q, Zhou Y, Tian H, Lin Y, Serov A, Zulevi B, Baca E, Fujimoto C, Chung H, Kim Y. Highly quaternized polystyrene ionomers for high performance anion exchange membrane water electrolysers. Nature Energy 2020;5(5):378-385.
[11] Yates J, Daiyan R, Patterson R, Amal R, Chang N. Techno-economic analysis of PV driven Hydrogen electrolysis - key drivers to economic feasibility. Australian PV Institute. 2019.

[12] Le Bideau D, Mandin P, Benbouzid M, Kim M, Sellier M. Review of necessary thermophysical properties and their sensivities with temperature and electrolyte mass fractions for alkaline water electrolysis multiphysics modelling. International Journal of Hydrogen Energy 2019;44(10):4553-4569.

[13] Solovey V, Zipunnikov M, Semikin V. Method for Calculating the Feed Water Replenishment Parameters under Electrolysis Process in Electrolyzer. FrenchUkrainian Journal of Chemistry 2020;8(2):168-175.

[14] Hamburg DYu, Dubovkin NF. Hydrogen. Properties, receipt, storage, transportation, application. Chemistry, 1989. $672 \mathrm{p}$.

[15] Solovei V, Kotenko A, Vorobiova I, Shevchenko A, Zipunnikov M. Basic Operation Principles and Control Algorithm for a High-pressure Membrane-less Electrolyser. Journal of Mechanical Engineering 2018;21(4):57-63.

[16] Solovey V, Shevchenko A, Zipunnikov M, Kotenko A, Khiem N, Tri B, Hai T. Development of high pressure membraneless alkaline electrolyzer. International Journal of Hydrogen Energy 2021. In Press. 